\title{
Ranking by weighted sum
}

\section{Tapan Mitra ${ }^{1} \cdot$ Kemal Ozbek ${ }^{2}$}

Received: 27 April 2019 / Accepted: 3 August 2020 / Published online: 9 August 2020

() The Author(s) 2020

\begin{abstract}
When choosing an alternative that has multiple attributes, it is common to form a weighted sum ranking. In this paper, we provide an axiomatic analysis of the weighted sum criterion using a general choice framework. We show that a preference order has a weak weighted sum representation if it satisfies three basic axioms: Monotonicity, Translation Invariance, and Substitutability. Further, these three axioms yield a strong weighted sum representation when the preference order satisfies a mild condition, which we call Partial Representability. A novel form of non-representable preference order shows that partial representability cannot be dispensed in establishing our strong representation result. We consider several related conditions each of which imply a partial representation, and therefore a strong weighted sum representation when combined with the three axioms. Unlike many available characterizations of weighted sums, our results directly construct a unique vector of weights from the preference order, which makes them useful for economic applications.
\end{abstract}

Keywords Decomposition of the set of irrationals - Partial representation . Substitutability $\cdot$ Weak representation $\cdot$ Weighted sum

JEL D01 $\cdot$ D60 $\cdot$ D70

\section{Introduction}

In many economic applications, choices are made by ranking alternatives based on their sum of weighted attributes. These weights typically reflect relative importance

\footnotetext{
Earlier versions of this work were circulated under the titles "On Representation and Weighted Utilitarian Representation of Preference Orders on Finite Streams" and "Weighted Utilitarianism over Finite Streams". Professor Tapan Mitra passed away on February 3, 2019. This final draft is dedicated to his memory.

$凶 \quad$ Kemal Ozbek

k.ozbek@soton.ac.uk

1 Goldwin Smith Professor of Economics, Cornell University, Ithaca, USA

2 Department of Economics, University of Southampton, University Road, Southampton S017 1BJ, UK
} 
and determine how much a unit of each attribute contributes to the weighted sum ranking. For instance, public policies may be compared by summing expert scores after weighing each expert's score by seniority; or political candidates can be ranked by summing items in their agendas after scaling each item by its urgency; or social plans may be ranked by summing individual payoffs after dividing each payoff by individual's wealth. In addition to its simplicity, what are the underlying common themes that justify weighted sum as a prevalent decision-making criterion? Relatedly, and perhaps more importantly, how can we elicit the weights, so as to make the weighted sum criterion more applicable in economic problems?

In this paper, using a general choice framework, we aim to shed some light on these questions by investigating the axiomatic foundations of the weighted sum. We take the primitive of our analysis as the preference order (i.e., a complete transitive binary relation) of a decision maker (DM, henceforth) over a set of alternatives $X$, where each alternative $x \in X$ represents a vector in the finite dimensional Euclidean space $\mathbb{R}^{n}$. Our objective is to identify testable implications of the weighted sum on the DM's preferences, as well as to elicit the weights associated with these preferences. In accomplishing these objectives, our approach is minimalistic; that is, we invoke relatively less demanding conditions induced by the weighted sum representation, which allow us to better clarify the role of each underlying principle.

We identify three basic implications of the weighted sum on the DM's preferences, namely Monotonicity, Translation Invariance, and Substitutability. Monotonicity requires that the DM be better off whenever every attribute of an alternative is improved. For instance, if each expert assigns a higher score to public plan $x$ than plan $y$, then the policy-maker strictly prefers $x$ over $y$. Translation Invariance means that in expressing the DM's preferences between two alternatives $x$ and $y$, account is taken only of how much each attribute changes. ${ }^{1}$ Thus, the preference between $x$ and $y$ in $X$ is the same as that between two other alternatives $x^{\prime}$ and $y^{\prime}$, if the change of each attribute is the same in both comparisons; that is, if $\left(x_{i}-y_{i}\right)=\left(x_{i}^{\prime}-y_{i}^{\prime}\right)$ for all $i \in\{1,2, \ldots, n\}$. For example, if the positions of two candidates shift equal amount for each item, then relative desirability of these candidates do not change. Substitutability, which is a key condition in our analysis, intuitively suggests that no attribute should be infinitely desirable relative to another attribute. In particular, Substitutability allows for a decrease in an attribute to be compensated by an increase in another attribute while keeping the DM indifferent. For instance, the social-planner remains indifferent between two actions when there is a certain payoff loss by an individual against a payoff gain by another individual while there is no change for other individuals.

The first main result of our analysis (Theorem 1) shows that if the DM's preferences satisfy the three axioms, then it must have a weak weighted sum representation. In particular, given the preference order, a vector $q$ of weights can be constructed by using the axiom of Substitutability. When the preference order also satisfies the Monotonicity and Translation Invariance axioms, we then show that whenever $q x>q y$, it must be the case that $x$ is strictly preferred to $y$. In fact, we show the weights $q$ of this weak representation must be unique. As such, except for the case when alternatives $x$ and

\footnotetext{
1 While we call this axiom [similar to Weibull (1985) and many others] Translation Invariance, it is also called Invariance with respect to individual origins of utilities [see, e.g., d'Aspremont and Gevers (1977)], Zero Independence [see, e.g., Moulin (1988)], or Linearity [see, e.g., Segal and Sobel (2002)].
} 
$y$ have exactly the same valuation (in terms of these unique weights $q$ ), the result provides an easily verifiable criterion for ranking the alternatives $x$ and $y .^{2}$

Given that the three axioms derive a weak representation for the order, a natural question is to ask if in fact they imply a strong weighted sum representation. That is, is it true that $q$ provides (in addition to a weak representation) a partial representation, so that $q x>q y$ whenever $x$ is strictly preferred over $y ?^{3}$ Our second main result shows that in general $q$ does not possess this property. Specifically, we construct an example of a novel preference order which satisfies the three axioms, but also allows for a strict preference between $x$ and $y$ even when $q x=q y$. As a result, an additional axiom is needed if one wants to ensure that the unique vector of weights $q$ yields a strong representation. ${ }^{4}$

Our third main result (Theorem 2) shows that when the preference order satisfying the three axioms has a partial representation (which can be in any form), the vector $q$ provides a partial representation, and therefore a strong weighted sum representation. Partial representability is a general property, which can be implied by many different conditions when supplied together with our three basic axioms. Regarding this, we consider several related conditions implied by the weighted sum representation (e.g., continuity, scale invariance, substitution, and anonymity) and discuss how alternative characterizations of the weighted sum representation can be established by using our main representation results.

Our way of obtaining weighted sum representation differs from available characterizations. While we construct the weights by linking them directly to the preference order, the weights are not constructed in the literature; only their existence is shown by employing some non-constructive methods. For instance, by appealing to a separating hyperplane argument, d'Aspremont and Gevers (2002, Theorem 4.1) establish that the Monotonicity and Translation Invariance axioms imply a weak weighted sum representation. Or using a functional equation argument, Segal and Sobel (2002, Lemma 4) show that the Monotonicity axiom and a weakening of the Translation Invariance axiom, together with a Separability and a Scale Invariance axiom imply a strong weighted sum representation. ${ }^{5}$ Constructing the weights of the representation can be quite important, however, especially when the objective is to apply the weighted sum

\footnotetext{
${ }^{2}$ Further, a strength of this result is that it relies only on intuitive axioms imposed on the preference order, and not on any technical continuity axioms, which are often used in the literature in obtaining representation results. For a discussion of partial characterizations of some other aggregation rules within the context of social choice theory, see Fleurbaey and Maniquet (2011). Other excellent surveys can be found in d'Aspremont and Gevers (2002) and Mongin and d'Aspremont (1998).

3 There are many related representation concepts used in the literature, such as Richter-Peleg representation, Aumann utility, quasi-representation, or weak utility. In particular, we use the term partial representation since we reserve "weak representation" for the converse implication. For a formal definition of partial representability, see Sect. 2.

4 Strong representation can be desirable in applications since when $q$ provides a partial representation (in addition to a weak representation), it can be used to find maximal elements of the preference order since in that case an alternative $x$ becomes a maximal element in a set if (and only if) it maximizes $q x$ over the given set.

5 Segal and Sobel (2002, Lemma 4)'s characterization of a strong weighted sum representation is logically equivalent to our Theorem 2. However, the axioms they use and the proof methods they apply substantially differ from ours. In particular, while we first construct a weak weighted sum representation from the three basic axioms, and then derive a strong representation by imposing partial representability,
} 
criterion in decision-making. In this regard, the Substitutability axiom allows us to directly elicit the weights from the preference order. Moreover, we show that these weights must be unique. As a result, our characterization results can be utilized with choice data when conducting policy relevant applications, as well as comparative statics exercises of the weighted sum representation.

There are also other important preferences within the general class considered by d'Aspremont and Gevers (2002). ${ }^{6}$ For instance, Hausner and Wendel (1952, Theorem 2.5) show that if a preference order satisfies, in addition to the Monotonicity and Translation Invariance axioms, the Scale Invariance axiom, then it has a "lexicographically ordered" weighted sum representation. Candeal (2013, Theorem 2) generalizes this result by dropping the Monotonicity axiom. Finally, d'Aspremont and Gevers (1977, Theorem 3) derive a weighted sum representation with equal weights by imposing an Anonymity axiom together with the Monotonicity, Translation Invariance, and Scale Invariance axioms. In addition to their practical relevance, our results help to clarify the relation of various weighted sum representations in this prior literature.

The rest of the paper is organized as follows. In Sect. 2, we introduce the framework and the three main axioms we use in our representation results. In Sect. 3, we show how to obtain the weights associated with a preference order, and provide our weighted sum representation results (weak and strong) using these weights. In this section, we also present our example of a preference order which satisfies the set of three basic axioms but fails to have a representation (and therefore in particular a weighted sum representation). Section 4 considers some related axioms and alternative characterizations of weighted sum representation, and we discuss how these characterizations follow from our results. Proofs of the main results (stated in Sect. 3) are provided in an "Appendix".

\section{Preliminaries}

Framework. Let $X$ denote the set of alternatives, which is an open convex subset of $\mathbb{R}^{n}$ (including the 0 vector) for some given $n \geq 2$, equipped with the usual topology. ${ }^{7}$ Let $I$ denote the set $\{1,2, \ldots, n\}$ and $J$ denote the set $\{2, \ldots, n\}$. For any $x \in X$ and $i, j \in I$ with $i \leq j$, let $x_{[i, j]} \in \mathbb{R}^{j-i+1}$ denote the vector $z \in \mathbb{R}^{j-i+1}$ such that $z_{k}=x_{i+k-1}$ for all $k=1, \ldots, j-i+1$. For any $x \in X$ and $i \in I$, let $x_{-i} \in \mathbb{R}^{n-1}$ denote the vector $z \in \mathbb{R}^{n-1}$ such that $z_{k}=x_{k}$ for all $k<i$ and $z_{k}=x_{k+1}$ for all $k \geq i$. For each $i \in I$, let $e^{i}$ be the $i$-th unit vector $(0, \ldots, 1, \ldots, 0)$ in $\mathbb{R}^{n}$.

Footnote 5 continued

Segal and Sobel (2002) first obtain a strong representation in an additively separable form, and then argue that this strong representation has to be a weighted sum.

${ }^{6}$ For an earlier account of related results on weak weighted sum representation, see Blackwell and Girshick (1954, Theorem 4.3.1), Roberts (1980, Theorem 2), Gevers (1979, Theorem 2) or d'Aspremont (1985, Theorem 3.3.3).

7 These alternatives could represent, for instance, environmental actions, which can have different degrees of endorsements from $n$-many experts; or they could represent political candidates, who may have certain positions on each of the $n$-many political issues; or they could denote vectors consisting of $n$-many people's payoffs associated with different social plans. 
Let $\Delta$ denote the $n-1$ simplex $\left\{q \in \mathbb{R}^{n}: q_{i} \geq 0\right.$ for all $i \in I$ and $\left.\sum_{i=1}^{n} q_{i}=1\right\}$, and $\Delta$ denote its interior. For any $x, y \in X$ write $x \asymp y$ if there exists some permutation $\sigma$ of $I$ such that $x_{i}=y_{\sigma(i)}$ for all $i \in I$. We denote by $\mathbb{N}$ the set of natural numbers, by $\mathbb{Q}$ the rationals in $\mathbb{R}$ (including 0 , by convention), and by $\mathbb{Q}_{++}$the subset of positive rationals. Finally, let $\mathbb{I}$ denote the set of irrational numbers in $\mathbb{R}$.

Representation. For any given binary relation $\succsim$ over $X$, let $\succ$ denote the asymmetric part, and $\sim$ denote the symmetric part. If there exist some $x, y \in X$ such that $x \succ y$ or $y \succ x$, we say $\succsim$ is non-trivial. For any $x, y \in X$, when $x \succ y$ or $y \succ x$ holds, we also write $x \nsim y$ and when neither $x \succsim y$ nor $y \succsim x$ holds, we may write $x \bowtie y$.

Let $\succsim$ be a non-trivial binary relation over $X$. We say that $\succsim$ has a weak representation if there exists a non-constant real valued function $v: X \rightarrow \mathbb{R}$ such that for all $x, y \in X, v(x)>v(y)$ implies $x \succ y$. We say that $v$ is a partial representation for $\succsim$ if for all $x, y \in X, x>y$ implies $v(x)>v(y)$. The function $v$ is called a strong representation of $\succsim$ if for all $x, y \in X, x \succ y$ if and only if $v(x)>v(y)$; that is, $v$ is a strong representation if it is both a weak and a partial representation. ${ }^{8}$

A (resp., weak; partial; strong) representation $v$ of $\succsim$ is called a (resp., weak; partial; strong) weighted sum representation if there exists some $q \in \Delta$ such that $v(x)=q x$ for all $x \in X$. We call such a $q \in \Delta$ a vector of weights and say that $q$ provides a (resp., weak; partial; strong) weighted sum representation for $\succsim$.

Given any alternative $x \in X$ and vector of weights $q \in \Delta$, let $L_{q}(x)$ denote the set of points in $X$ whose weighted sum with respect to $q$ is equal to $q x$; that is, $L_{q}(x)=\{y \in X: q y=q x\}$. Finally, given any $x \in X$ and binary relation $\succsim$ over $X$, let $\mathcal{I}_{\succsim}(x)$ denote the indifference curve passing through the alternative $x \in X$; that is, $\mathcal{I}_{\succsim}(\tilde{x})=\{y \in X: y \sim x\}$.

Examples. In order to clarify the nature of different representation concepts defined above, consider the following examples of binary relations, where each one of them lacks either a weak or a partial representation, and therefore a strong representation. ${ }^{9}$

Example 1 For all $x, y \in X$, let $x \succsim y$ if $x \geq_{L} y$, where $\geq_{L}$ denotes the lexicographic order on $\mathbb{R}^{n}$. We claim that $\succsim$ has a weak representation, but it has no partial representation. To see this, note that whenever $x_{1}>y_{1}$, by definition $x \succ y$. Hence, $v(x)=x_{1}$ for all $x \in X$ is a weak (weighted sum) representation for $\succsim$. Now assume, for contradiction, that there is some partial representation $u: X \rightarrow \mathbb{R}$ for $\succsim$; that is, $x \succ y$ implies $u(x)>u(y)$ for all $x, y \in X$. Notice that $u(x)$ must be independent of any $x_{j}$ for $j \neq 1$ since whenever $x_{1}>y_{1}$, then $u(x)>u(y)$ regardless of $x_{-1}$ and $y_{-1}$. In that case, however, we must have $u(x)=u(y)$ whenever $x_{1}=y_{1}$, but also $u(x) \neq u(y)$ whenever $x \neq y$, a contradiction.

Example 2 For all $x, y \in X$, let $x \succsim y$ if $x \geq_{D} \quad y$, where $\geq_{D}$ denotes the vector dominance on $\mathbb{R}^{n}$. We claim that $\succsim$ has a partial representation, but it has no weak representation. To see this, note that whenever $x>y, \sum_{i \in I} x_{i}>\sum_{i \in I} y_{i}$. Hence, $v(x)=\sum_{i \in I} x_{i}$ for all $x \in X$ is a partial (weighted sum) representation for $\succsim$. Now

\footnotetext{
8 Whenever $\succsim$ is a complete binary relation, function $v$ becomes a strong representation for $\succsim$ if for all $x, y \in X, x \succsim y$ if and only if $v(x) \geq v(y)$.

9 For an example of a binary relation that has a weak and a partial representation, but has no strong representation, see Footnote 18.
} 
assume, for contradiction, that there is some weak representation $u: X \rightarrow \mathbb{R}$ for $\succsim$; that is, there exists a non-constant function $u$ such that $u(x)>u(y)$ implies $x \succ y$ for all $x, y \in X$. Notice that whenever $x \succ y$, then $u(x) \geq u(y)$ and whenever $x \sim y$ or $x \bowtie y$, then $u(x)=u(y)$. Let $x, y \in X$ such that $x \succ y$. By definition, there must exist some $i \in I$ such that $x_{i}>y_{i}$ and $x_{j} \geq y_{j}$ for all $j \neq i$. Let $z \in X$ such that $y_{i}>z_{i}$ and $z_{j}>x_{j}$ for all $j \neq i$. Then we have $x \bowtie z$ and $y \bowtie z$, and so $u(x)=u(z)$ and $u(y)=u(z)$ implying that $u$ must be a constant function, a contradiction.

Example 3 For all $x, y \in X$, let $x \succsim y$ if $x_{[1, k]} \geq_{L} \quad y_{[1, k]}$ and $x_{[k+1, n]} \geq_{D} y_{[k+1, n]}$ where $1<k<n$, while $\geq_{L}$ denotes the lexicographic order on $\mathbb{R}^{k}$ and $\geq_{D}$ denotes the vector dominance on $\mathbb{R}^{n-k}$. Then, $\succsim$ has neither a partial representation nor a weak representation since the impossibility arguments given in Examples 1 and 2 both apply in this case.

Basic axioms. The primitive of our analysis is a binary relation $\succsim$ over $X$, which we assume to be a preference order (i.e., a complete and transitive binary relation) indicating the DM's preferences. It is straightforward to verify that a strong weighted sum representation with positive weights induces the following three axioms on the DM's preferences.

Axiom 1 (Monotonicity) For all $x, y \in X$, if $x_{i}>y_{i}$ for all $i \in I$, then $x \succ y$.

Axiom 2 (Translation invariance) For all $x, y, z \in X$ with $x+z, y+z \in X$, if $x \succsim y$, then $x+z \succsim y+z$.

Axiom 3 (Substitutability) For all $i, j \in I$ and $x \in X$, there exists $y \in X$ such that $x_{i}>y_{i}, x_{j}<y_{j}, x_{k}=y_{k}$ for all $k \in I \backslash\{i, j\}$, and $x \sim y$.

When the DM forms preferences for binary comparisons of alternatives, Monotonicity expresses that more of each attribute leads to a better alternative, while Translation Invariance means that not absolute, but relative values of attributes matter and Substitutability says that it is possible to exchange each attribute with any other attribute.

Notice that the binary relations defined in Examples 1-3, all of which have no strong representation, satisfy Axioms 1 and 2, but not Axiom $3 .{ }^{10}$ In the next section, we will analyze the implications of imposing Axiom 3 in addition to Axioms 1 and 2 in terms of representability of the DM's preferences by a weighted sum.

\section{Analysis}

This section contains our main results on weighted sums; a weak representation result, a counter example on representability and a strong representation result.

\footnotetext{
10 If a complete transitive binary relation satisfies Monotonicity, then it must have a weak representation [see Mitra and Ozbek (2013, Proposition 1)]. As such, to illustrate some examples of a monotone relation with no weak representation, in Examples 2 and 3 above, we consider transitive, but incomplete binary relations.
} 


\subsection{Weights of the attributes}

Using the axiom of Substitutability (Axiom 3), we can associate the DM's preference order $\succsim$ directly with a vector of weights $q \in \dot{\Delta}$.

To see this, note that by Substitutability, we can find for each $j \in J$, some $\alpha_{j}, \beta_{j} \in$ $\mathbb{R}_{++}$, such that

$$
0 \sim \alpha_{j} e^{1}-\beta_{j} e^{j}
$$

Define $\gamma_{1}=1$, and for each $j \in J$, define $\gamma_{j} \equiv\left(\alpha_{j} / \beta_{j}\right)$. Then, denoting $\left(\gamma_{1}+\right.$ $\left.\cdots+\gamma_{n}\right)$ by $\sigma$, we see that $q \in \stackrel{\Delta}{ }$, where:

$$
q=\left(\gamma_{i} / \sigma\right), \quad \forall i \in I
$$

We will show that these weights will be precisely the weights placed on the attributes of the alternatives in the representation, when the preference order has a weighted sum representation.

\subsection{Weak representation by a weighted sum}

We now show that the vector $q$ defined in Eq. (2) provides an easily verifiable criterion for ranking alternatives in $X$. Specifically, Theorem 1 establishes that whenever the DM's preference order satisfies Axioms 1-3, then any two alternatives can be directly ranked by using $q$ as long as these two alternatives do not provide exactly the same valuation in terms of $q$.

Theorem 1 Let $\succsim$ be a preference order satisfying Axioms $1-3$. Then, for all $x, y \in X$, $q x>q y$ implies $x \succ y$. Moreover, $q$ is the unique vector in $\triangle$ providing such a weak representation for $\succsim$.

Our way of obtaining a weak weighted sum representation differs from available characterizations since we directly link the weights to the preference order. ${ }^{11}$ In the available characterizations, rather than constructing the weights, only their existence is shown by invoking a non-constructive method. ${ }^{12}$ By contrast, we construct the weights uniquely allowing for policy motivated applications, as well as related comparative statics analysis using choice data.

\footnotetext{
11 One might think that the proof of Theorem 1 could easily follow from a standard separation argument, where each point is separated from its strict upper (or lower) contour set with a hyperplane. However, as we demonstrate in Sect. 3.3, this is not necessarily the case in our setting. The reason is that, since we impose weaker axioms, the strict contour sets of the preference order $\succsim$ may not be open or convex. As a result, to establish the form of separation given in Theorem 1, one needs to consider a different proof method. In this regard, we first construct the weights in $q$ by Axiom 3 and then directly show that the sets $\{z \in X: q z>0\}$ and $\{z \in X: 0 \succsim z\}$ must be disjoint by also using Axioms 1 and 2. As such, since our proof method has relatively less requirements, it can be applied in many more general choice settings.

12 These methods typically concern the existence of separating hyperplanes, functional equation solutions, or supremum/infimum points. See, for instance, d'Aspremont and Gevers (2002, Theorem 4.1), Segal and Sobel (2002, Lemma 4), or Hausner and Wendel (1952, Theorem 2.5) utilizing such methods.
} 
To see that $q$ is the only vector in $\Delta$ providing a weak representation, suppose on the contrary there is some $p \in \Delta$ with $p \neq q$, which also provides a weak weighted sum representation for the preference order; that is, assume that for all $x, y \in X$, we have $p x>p y$ implies $x \succ y$. In that case, clearly we can find some $i, j \in I$ such that $q_{i}>p_{i}$ and $q_{j}<p_{j}$. Note then that $r \equiv q_{i} p_{j}-q_{j} p_{i}>0$, and define $x \in X$ as $x_{i}=\frac{\left(p_{j}+q_{j}\right)}{r}, x_{j}=-\frac{\left(p_{i}+q_{i}\right)}{r}, x_{k}=0$ for all $k \in I \backslash\{i, j\}$ and $y=0$. Then, $q x=1$ while $p x=-1$, while $q y=p y=0$. Thus, $q x>q y$ and so $x>y$ and further $p x<p y$ so that $y>x$, a contradiction.

\subsection{A preference order with no partial representation}

Theorem 1 shows that whenever the DM's preference order $\succsim$ satisfies the three axioms (Axioms 1-3), the unique vector $q$ obtained in Eq. (2) yields a weak weighted sum representation. However, does this necessarily imply that $q$ should also yield a partial representation, and therefore a strong representation when the three basic axioms hold? That is, is it also true that $q x>q y$ whenever $x$ is strictly preferred over $y$ ? Our second main result provides a negative answer to this question by showing that a preference order that satisfies the three axioms, but has no partial representation can be constructed.

To gain some intuition on why the three basic axioms are not enough to guarantee a partial representation for the preference order, consider the sets $L_{q}(x)$ and $\mathcal{I}_{\succ}(x)$ for some given $x \in X$, where $q$ is the unique vector defined in Eq. (2). Notice that whenever the preference order has a strong weighted sum representation, then the set $L_{q}(x)$ must coincide with the indifference curve $\mathcal{I}_{\succsim}(x)$. Theorem 1 shows that whenever the order satisfies Axioms 1-3, then $\mathcal{I}_{\succsim}(x) \subset L_{q}(x)$. ${ }^{13}$ However, Axioms 1-3 do not necessarily guarantee that $L_{q}(x) \subset \mathcal{I}_{\succsim} \widetilde{(x)}$ also holds. That is, there can be many $y \in L_{q}(x)$ such that $y$ is not indifferent to $x$, and so $y \notin \mathcal{I}_{\succ}(x)$. In other words, indifference curves of a preference order satisfying Axioms $1-\widetilde{3}$ can have arbitrarily many gaps in them. Our example below shows that it is indeed for this very reason that a preference order satisfying all three basic axioms may not have a partial representation at all.

Definition of the preference order. Let $\succsim$ be a binary relation on $X=\mathbb{R}^{n}$ which satisfies for all $x, y \in \mathbb{R}^{n}$,

$$
x \succsim y \text { if }\left(\sum_{i=1}^{n}\left(x_{i}-y_{i}\right), \phi\left(x_{1}-y_{1}\right)\right) \geq_{L}(0,0)
$$

where $\geq_{L}$ is the lexicographic order defined on $\mathbb{R}^{2}$ and $\phi($.$) is an indicator function$ on $\mathbb{R}$ defined as: $\varphi(r)=1$ if $r \in \mathbb{A}, \varphi(r)=0$ if $r \in \mathbb{Q}$, and $\varphi(r)=-1$ if $r \in \mathbb{B}$, where $\mathbb{A}$ and $\mathbb{B}$ are subsets of the set of irrationals $\mathbb{I}$ with the following four properties:

\footnotetext{
13 Since $\succsim$ is complete, Theorem 1 shows that $x \succsim y$ implies $q x \geq q y$ for all $x, y \in X$, which in turn implies $\mathcal{I}_{\succsim}(x) \subset L_{q}(x)$ for all $x \in X$.
} 
(i) $\mathbb{A} \cap \mathbb{B}=\emptyset$ and $\mathbb{A} \cup \mathbb{B}=\mathbb{I}$, (ii) $\mathbb{A}=-\mathbb{B}$, (iii) $a, a^{\prime} \in \mathbb{A}$ implies $a+a^{\prime} \in \mathbb{A}$, and (iv) $a \in \mathbb{A}$ and $r \in \mathbb{Q}$ implies $a+r \in \mathbb{A}$. ${ }^{14}$

Notice that the lexicographic order, which is the pre-eminent example of nonrepresentability of a preference order (since Debreu (1954)), satisfies the Monotonicity and Translation Invariance axioms, but it violates the Substitutability axiom, since it does not allow for any substitution possibilities. By contrast, the preference order we construct in Eq. (3) satisfies all three of these axioms (and therefore allows for substitutions), yet it has no partial representation, and therefore it has no strong representation. We believe this novel finding can be useful in deriving similar impossibility results. ${ }^{15}$ Verifying the axioms. Clearly, $\succsim$ is complete. We check transitivity of $\succsim$ as follows. For any $x, y, z \in X$ with $x \succsim y$ and $y \succsim z$, we have $\sum_{i \in I} x_{i} \geq \sum_{i \in I} y_{i}$ and $\sum_{i \in I} y_{i} \geq \sum_{i \in I} z_{i}$. Thus, we must have $\sum_{i \in I} x_{i} \geq \sum_{i \in I} z_{i}$. If $\sum_{i \in I} x_{i}>\sum_{i \in I} z_{i}$, then by Eq. (3) we have $x \succsim z$. If $\sum_{i \in I} x_{i}=\sum_{i \in I} z_{i}$, then both $\left(x_{1}-y_{1}\right)$ and $\left(y_{1}-z_{1}\right)$ must belong to $\mathbb{A} \cup \mathbb{Q}$ by Eq. (3) and so, $\left(x_{1}-z_{1}\right) \in \mathbb{A} \cup \mathbb{Q}$, showing that $x \succsim z$. Hence, $\succsim$ is a preference order. Let $x, y \in X$ such that $x_{i}>y_{i}$ for all $i \in I$. Then $\sum_{i \in I} x_{i}>$ $\sum_{i \in I} y_{i}$ and so we must have $x \succ y$ by Eq. (3). Thus, $\succsim$ satisfies Axiom 1 . For any $x, y, z \in X, \sum_{i \in I} x_{i} \geq \sum_{i \in I} y_{i}$ is equivalent to $\sum_{i \in I}\left(x_{i}+z_{i}\right) \geq \sum_{i \in I}\left(y_{i}+z_{i}\right)$. We also have $\phi\left(x_{1}-y_{1}\right)=\phi\left(\left(x_{1}+z_{1}\right)-\left(y_{1}+z_{1}\right)\right)$. Therefore by Eq. (3), $x \succsim y$ if and only if $x+z \succsim y+z$, showing that $\succsim$ satisfies Axiom 2. For any $i, j \in I$, with $i \neq j$, and $x \in X$, let $y \in X$ be such that $y_{i}=x_{i}-\epsilon, y_{j}=x_{j}+\epsilon$ for some $\epsilon \in \mathbb{Q}_{++}$and $y_{k}=x_{k}$ for every $k \in I \backslash\{i, j\}$. We have $\sum_{r \in I} x_{r}=\sum_{r \in I} y_{r}$ and $x_{1}-y_{1} \in \mathbb{Q}$. Thus by Eq. (3), $x \sim y$ establishing that $\succsim$ satisfies Axiom 3 .

No partial representation. Clearly, the vector $q=(1 / n) \in \Delta$ provides a weak representation for $\succsim$. We now claim that $\succsim$ has no partial representation. Suppose, for contradiction, that there exists a real-valued function $v: X \rightarrow \mathbb{R}$ which yields a partial representation for $\succsim$. Associate with each pair of numbers $(c, d) \in \mathbb{R}^{2}$, a non-empty subset of $X, D(c, d)=\left\{x \in X: \sum_{i \in I} x_{i}=c\right.$ and $\left.x_{1}=d\right\}$. Fix two numbers $d_{1}, d_{2} \in \mathbb{R}$ with $\left(d_{2}-d_{1}\right) \in \mathbb{A}$. Given any $c \in \mathbb{R}$, pick a unique element $g(c)$ from $D\left(c, d_{1}\right)$ and a unique element $h(c)$ from $D\left(c, d_{2}\right)$, by using the Axiom of Choice. Define $\alpha(c)=v(g(c))$ and $\beta(c)=v(h(c))$. It follows from Eq. (3) that $h(c) \succ g(c)$ for every $c \in \mathbb{R}$, and $g\left(c^{\prime}\right) \succ h(c)$ whenever $c, c^{\prime} \in \mathbb{R}$ and $c^{\prime}>c$. Thus, we have (i) $\alpha(c)<\beta(c)$ for every $c \in \mathbb{R}$, and (ii) $\beta(c)<\alpha\left(c^{\prime}\right)$ for all $c, c^{\prime} \in \mathbb{R}$ satisfying $c<c^{\prime}$. Define for all $c \in \mathbb{R}$, the interval $E(c)=[\alpha(c), \beta(c)]$. Then, whenever $c, c^{\prime} \in \mathbb{R}$ with $c \neq c^{\prime}$, we must have $E(c)$ to be disjoint from $E\left(c^{\prime}\right)$. Thus, there is a one to one correspondence between the set of real numbers (which is uncountable) and a set of non-degenerate pairwise disjoint intervals (which is countable), a contradiction, establishing our claim.

\footnotetext{
14 For the existence of this particular decomposition of the set irrational numbers, see Theorem 3 in "Appendix A.3".

15 For an account of the literature, and for the key references on the representability of preference orderings, see, e.g., Bridges and Mehta (1995), Mehta (1998), and Banerjee and Mitra (2018).
} 


\subsection{Strong representation by a weighted sum}

In this section, we turn to the issue of providing a strong representation for the preference order satisfying the three axioms.

The counter example given in Eq. (3) demonstrates that the set of three axioms is not sufficient to yield a partial representation for the preference order. This naturally raises the following question: if a preference order has a partial representation and satisfies Axioms 1-3, does the order necessarily have a strong weighted sum representation? The answer to this question turns out to be affirmative as shown by the following result.

Theorem 2 Let $\succsim$ be a preference order on $X$ with a partial representation. If $\succsim$ satisfies Axioms 1-3, then the unique vector $q$ provides a strong weighted sum representation for $\succsim$; that is, $x \succsim y$ if and only if $q x \geq q y$.

In establishing Theorem 2, we first note that whenever the preference order $\succsim$ has some partial representation $v$ (which can be in any form), then Axioms 1-3 imply that the vector of weights $q$ defined in Eq. (2) provides a partial representation too; that is, for all $x, y \in X, x \succ y$ implies $q x>q y .{ }^{16}$ Since we know by Theorem 1 that under these three axioms $q$ already yields a weak representation and therefore $q x>q y$ implies $x \succ y$, we then derive a strong weighted sum representation with vector $q .{ }^{17}$

Theorem 2 suggests that one way of obtaining a strong weighted sum representation for a given preference order (satisfying Axioms 1-3) is to add an axiom to ensure that a partial representation for the preference order exists. ${ }^{18}$ In the next section, we consider several axioms which serve this purpose.

\section{Related axioms and characterizations}

In this section, we consider some related axioms (which are all implied by the weighted sum representation) and discuss how alternative characterizations of the weighted sum can be obtained by using our main results.

\footnotetext{
16 Since $\succsim$ is a complete binary relation, $q$ becomes a partial representation if and only if for all $x, y \in X$, $q x=q y$ implies $x \sim y$; that is, whenever $L_{q}(x) \subset \mathcal{I}_{\succ}(x)$ for all $x \in X$. This means a preference order $\succsim$, which has a partial representation and satisfies Axioms 1-3, cannot have gaps in its indifference curves.

17 Note that if a given binary relation $\succsim$ has some weak representation $v_{1}: X \rightarrow \mathbb{R}$ and partial representation $v_{2}: X \rightarrow \mathbb{R}$, then there must exist a weakly increasing function $f: \mathbb{R} \rightarrow \mathbb{R}$ such that $v_{1}(x)=f\left(v_{2}(x)\right)$ for all $x \in X$. As such, Theorem 2 shows that whenever the preference order $\succsim$ satisfies Axioms $1-3$, then $f$ can be taken as the identity map, which is strictly increasing.

18 If a given binary relation $\succsim$ has a weak representation $v_{1}$ and a partial representation $v_{2}$ such that $v_{1}(x)=f\left(v_{2}(x)\right)$ for some strictly increasing function $f: \mathbb{R} \rightarrow \mathbb{R}$, then clearly both $v_{1}$ and $v_{2}$ must provide a strong representation for $\succsim$. In general, however, $f$ may not be strictly increasing. In such cases, it is possible to have the underlying binary relation $\succsim$ without a strong representation. For instance, this is the case for the preference relation $\succsim$ defined for all $x, y \in X$ by $x \succsim y$ if $f\left(\sum x_{i}\right)>f\left(\sum y_{i}\right)$ or $\left[f\left(\sum x_{i}\right)=f\left(\sum y_{i}\right)\right.$ and $\left.x \geq_{D} y\right]$, where $f$ is the floor function (i.e., the rounding down function) and $\geq_{D}$ denotes the vector dominance on $\mathbb{R}^{n}$. Here $v_{1}(x)=f\left(\sum x_{i}\right)$ is a weak representation and $v_{2}(x)=\sum x_{i}$ is a partial representation for $\succsim$, but clearly this binary relation has no strong representation.
} 


\subsection{Continuity}

A weak continuity axiom for the preference order can be given as follows.

Axiom 4 (Archimedean) For all $x, y \in X$, if $x \succ 0$ and $y \succ 0$, then $\lambda x>y$ for some $\lambda \in \mathbb{R}_{++}$.

Imposing the Archimedean axiom allows us to rule out non-representable preferences satisfying Axioms 1-3 (e.g., the preference order given in Eq. (3)). ${ }^{19}$ In fact, if $\succsim$ satisfies Axiom 4 (in addition to Axioms 1-3), then the vector $q$ constructed in Eq. (2) must yield a partial representation for $\succsim$; that is, $q x>q y$ whenever $x \succ y$. $^{20}$

Corollary 1 Let $\succsim$ be a preference order on X satisfying Axioms 1-3 and Axiom 4. Then, for all $x, y \in X, x \succsim y$ if and only if $q x \geq q y$.

To see that $q$ provides a partial representation for $\succsim$, assume on the contrary that we could find some $x, y \in X$ with $x>y$ and $q x \leq q y$. In that case, $q x=q y$ by Theorem 1, and so for any $z \in X$ with $q z>0$ we have $q z>q(x-y)=0$ implying $z \succ \lambda(x-y)$ for all $\lambda \in \mathbb{R}_{++}$. But then since $z \succ 0$ by Theorem 1 and $x-y \succ 0$ by Axiom 2, we have a violation of Axiom 4 .

\subsection{Scale invariance}

An invariance axiom with respect to scale can be given as follows.

Axiom 5 (Scale invariance) For all $x, y \in X$ and $\lambda \in \mathbb{R}_{++}$with $\lambda x, \lambda y \in X$, if $x \succsim y$ then $\lambda x \succsim \lambda y$.

Hausner and Wendel (1952) show that whenever the preference order $\succsim$ satisfies Monotonicity (Axiom 1), Translation Invariance (Axiom 2) and Scale Invariance (Axiom 5), the preference order has a lexicographically ordered weighted sum representation. ${ }^{21}$ That is, there exists a multi-valued real function $V: X \rightarrow \mathbb{R}^{n}$ such that for all $x, y \in X, x \succsim y$ if and only if $V(x) \geq_{L} V(y)$, where for all $i \in I, V_{i}(x)=q^{i} x$ for some $q^{i} \in \Delta$ and $\geq_{L}$ is the lexicographic binary relation defined over $\mathbb{R}^{n} .^{22}$

\footnotetext{
${ }^{19}$ By contrast, there are a number of results in the literature which derive weighted sum representations by using stronger forms of continuity (closed upper or lower contour sets) together with Translation Invariance, while dispensing Monotonicity and Substitutability altogether; see, for instance, Trockel (1992), Candeal and Indurain (1995), Neuefeind and Trockel (1995).

20 Other weak continuity axioms, which are implied by the strong weighted sum representation and which can imply partial representation for monotone preferences, are Scalar Continuity [see Mitra and Ozbek (2013)] or Wold Condition [see Banerjee and Mitra (2018)].

${ }^{21}$ For the statement of Hausner and Wendel (1952)'s result in the context of expected utility theory, see Hara et al. (2019). See also Birkoff (1948, p. 240) and Krause (1995, Theorem 2) who use a vector basis argument for the characterization of lexicographically ordered weighted sum preferences.

22 Intuitively, the DM first uses the weights in $q^{1} \in \Delta$ as a weak representation; if $q^{1} x=q^{1} y$ for some $x, y \in X$, the DM then uses the weights in $q^{2}$ to rank $x$ and $y$; if, however, it is the case that $q^{2} x=q^{2} y$, the DM then uses the weights in $q^{3}$ to rank $x$ and $y$ and so on.
} 
Clearly, when we add the Substitutability axiom, we must have $q^{i}=q^{j}$ for all

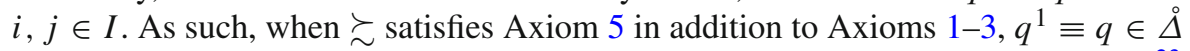
yields a strong weighted sum representation for $\succsim$ as stated in the following result. ${ }^{23}$

Corollary 2 Let $\succsim$ be a preference order on $X$ satisfying Axioms 1-3 and Axiom 5. Then, for all $x, y \in X, x \succsim y$ if and only if $q x \geq q y$.

Corollary 2 shows that whenever the DM's preference order $\succsim$ satisfies Axiom 5, the vector $q$ constructed in Eq. (2) provides not only a weak, but also a partial representation for $\succsim .{ }^{24}$ By contrast, to derive the existence of weights Hausner and Wendel (1952, Theorem 2.5) use a supremum argument, which makes their result non-constructive. We also note that Hausner and Wendel (1952) obtain their characterization of weighted sum by imposing the Archimedean axiom (Axiom 4) instead of Substitutability (Axiom 3). ${ }^{25}$

\subsection{Substitutability}

The Substitutability axiom, by which we directly obtain the vector of weights $q$ (as defined in Eq. (2)), is crucial for our study of weighted sum representations. To better understand its role in our analysis, consider the following list of five related conditions which are all implied by the strong weighted sum representation with positive weights.

1. (S1) For all $x \in X$, there exists $y \in \mathcal{I}_{\succsim}(x)$ such that $x \neq y$.

2. (S2) For all $x \in X$, there exists $y \in \widetilde{\mathcal{I}_{\succsim}}(x)$ such that $y=x-a e^{i}+b e^{j}$ for some $i, j \in I$ with $i \neq j$ and $a, b>0$.

3. (S3) For all $x \in X$, there exists $y \in \mathcal{I}_{\succsim}(x)$ for each $i \in I$ such that $y=x-a e^{i}+b e^{j}$ for some $j \in I$ with $i \neq j$ and $a, b \stackrel{\sim}{>} 0$.

4. (S4) For all $x \in X$, there exists $y \in \mathcal{I}_{\succsim}(x)$ for each $i, j \in I$ with $i \neq j$ such that $y=x-a e^{i}+b e^{j}$ for some $a, b>0$.

5. (S5) For all $x \in X$, there exists $y \in \mathcal{I}_{\succsim}(x)$ for each $i, j \in I$ with $i \neq j$ and $a>0$ such that $y=x-a e^{i}+b e^{j}$ for some $b>0$.

23 In a related work, Yoshihara and Veneziani (2018) consider evaluation of labor content, and characterize the weighted sum of labor amounts using axioms formally similar to Monotonicity, Substitutability (which they call Labor Trade-offs), and a consistency requirement named Mixture Invariance (which is equivalent to a combination of Translation Invariance and Scale Invariance). Our proof method differs from theirs since we first obtain a weak representation.

24 To see this more directly, note that whenever $q x=q y$ for $x, y \in X$, we can find some $\left(\lambda_{2}, \ldots, \lambda_{n}\right) \in \mathbb{R}^{J}$ such that $y=x+\sum_{j=2}^{n} \lambda_{j}\left(\alpha_{j} e^{1}-\beta_{j} e^{j}\right)$, where $\alpha_{j}, \beta_{j}$ are defined as in Eq. (1). Thus, by iterative application of Axioms 5 and 2, we obtain $x \sim y$ showing that $q$ provides a partial representation for $\succsim$.

25 Relatedly, by appealing to the Axiom of Choice, Chipman (1960, Theorem 3.2) obtains a lexicographically ordered multi-valued real representation $f: X \rightarrow \mathbb{R}^{\Omega}$ for a given preference order $\succsim$ defined over an abstract choice space $X$. Moreover, Chipman (1960, Theorem 3.4) shows that $f$ must provide a real-valued representation for $\succsim$ whenever $\succsim$ satisfies two additional axioms, which he calls "Axiom of Density" and "Axiom of Substitution". While Axiom of Density is a richness condition for the choice space $X$ with respect to the given order $\succsim$, Axiom of Substitution is essentially a continuity property for the preference order $\succsim$ over $X$ requiring $X$ to be connected with respect to a topology induced by $f$; in fact, Chipman (1960, Theorem 3.5) shows that Axiom of Substitution is equivalent to an Archimedean property. By contrast, our choice space $X$ is rich enough and our Substitutability axiom is not a continuity property since, for instance, the preference order $\succsim$ defined in Eq. (3) satisfies Substitutability, but obviously fails to satisfy any continuity property. 
Condition (S4) is a re-formulation of Substitutability (Axiom 3). Notice also that conditions above are given in an increasing order of strength. In particular, (S1) is the weakest while (S5) is the strongest condition. Since (S1) and (S2) are weaker than other substitution conditions, they can accommodate many preference orders that the stronger substitution conditions do not permit.

Example 4 Let $1 \leq k \leq n$ and define $\succsim$ over $X$ such that for all $x, y \in X, x \succsim y$ if $x_{[1, k]} \geq_{L} y_{[1, k]}$, where $\geq_{L}$ is the lexicographic order over $\mathbb{R}^{k}$. Then, clearly $\succsim$ is a preference order which satisfies Axioms 1 and 2 . Moreover, $\succsim$ can satisfy (S1) or (S2), but not other substitution conditions listed above. In particular, if (S1) (resp., (S2)) holds, then $k \leq n-1$ (resp., $k \leq n-2$ ) to allow for indifferences between alternatives. However, these indifferences hold only due to the insensitivity the order $\succsim$ exhibits towards some attributes, and not due to any substitutability between these attributes.

In view of Example 4, we see that (S1) or (S2)'s plausibility as a substitution condition is questionable. Now consider a related preference order $\succsim$ which can satisfy any of the substitution conditions listed above.

Example 5 Let $1 \leq m \leq k \leq n$ and define $\succsim$ over $X$ such that for all $x, y \in X, x \succsim y$ if $\left(\sum_{i=1}^{m} x_{i} / k, \sum_{i=m+1}^{k} x_{i} / k\right) \geq_{L}\left(\sum_{i=1}^{m} y_{i} / k, \sum_{i=m+1}^{k} y_{i} / k\right)$, where $\geq_{L}$ is the lexicographic order over $\mathbb{R}^{2}$. Then, clearly $\succsim$ is a preference order which satisfies Axioms 1 and 2. Moreover, if (S3) holds, then $m \geq 2$ (with $k \geq m+2$ if already $k>m$ ). Also notice that when (S3) holds, it is possible to have $k \leq n-2$, and so $\succsim$ can exhibit indifferences due to the insensitivity towards some attributes. As such, plausibility of (S3) as a substitution condition is questionable. On the other hand, when the preference order $\succsim$ satisfies at least (S4), insensitivity towards attributes cannot happen since in this case it has to be $k=n$. Moreover, when (S4) holds, any two attributes $i, j \in I$ must be substitutable, and so $m=k$ yielding a strong weighted sum representation.

We see from Examples 4 and 5 that in order to guarantee substitutability between all attributes, at least condition (S4) should be imposed. Finally, note that since (S5) is a stronger condition than (S4), there may be preferences which satisfy condition (S4), but which are incompatible with condition (S5). For instance, the preference order $\succsim$ defined by Eq. (3) satisfies Axioms 1 and 2, and condition (S4), but violates condition (S5). To see this, let $x \in X$ and $a \in \mathbb{I}$. Then $\varphi(a) \neq 0$ leading to $x \nsim x-a e^{1}+b e^{2}$ for any $b \in \mathbb{R}$. In fact, the following result shows that condition (S5) can eliminate all non-representable orders satisfying Axioms 1 and 2. ${ }^{26}$

Corollary 3 Let $\succsim$ be a preference order on $X$ satisfying Axioms 1 and 2, and Condition (S5). Then, for all $x, y \in X, x \succsim y$ if and only if $q x \geq q y$.

Corollary 3 shows that in conjunction with Axioms 1 and 2, condition (S5) implies that the preference order $\succsim$ has a strong representation given by the vector of weights

\footnotetext{
26 In general, substitutability, even in the weakest form of condition (S1), is not necessary for representability. This is the case, for instance, when the preference order has a representation and all of its indifference sets are singletons [see, e.g., Banerjee and Mitra (2018, Example 3)].
} 
$q$ derived in Eq. (2). The fact that $q$ provides a weak representation for $\succsim$ directly follows from Theorem 1 since condition (S5) allows for a wider range of substitutions than condition (S4) (or equivalently Axiom 3) does. To see that $q$ also yields a partial representation for $\succsim$ in this case, let $x, y \in X$ such that $q x=q y$ and $x \neq y$. For simplicity, assume that $x_{i}>y_{i}$ and $x_{j}<y_{j}$ for some $i, j \in I$, and $x_{k}=y_{k}$ for all $k \neq i, j$. Notice that we can write $y$ as $y=x-\left(x_{i}-y_{i}\right) e^{i}+\left(y_{j}-x_{j}\right) e^{j}$ in this case. ${ }^{27}$ By condition (S5), there must exist some $b>0$ such that $x \sim z \equiv x-\left(x_{i}-y_{i}\right) e^{i}+b e^{j}$. Since $q$ is the unique vector of weights providing a weak representation for $\succsim$, we have $\frac{q_{i}}{q_{j}}=\frac{b}{x_{i}-y_{i}}$. As a result, we obtain $b=\frac{q_{i}}{q_{j}}\left(x_{i}-y_{i}\right)$, and so $q z=q x$ implying that $y=z$. As such, we must have $x \sim y$ showing that $q$ yields a partial representation, and therefore a strong weighted sum representation for $\succsim .{ }^{28}$

\subsection{Anonymity}

A form of equity axiom, which expresses equal treatment of attributes, is known as the Anonymity axiom.

Axiom 6 (Anonymity) For all $x, y \in \mathbb{R}^{n}$, if $x \asymp y$, then $x \sim y$.

Anonymity can be used to characterize the weighted sum representation with equal weights; that is, $q_{i}=1 / n$ for all $i \in I .^{29}$

Corollary 4 Let $\succsim$ be a preference order on $X$ satisfying Axioms 1-2 and Axiom 6. Then, for all $x, y \in X, x \succsim y$ if and only if $q x \geq q y$, where $q_{i}=1 / n$ for all $i \in I$.

To see this, note that Axiom 6 directly implies the argument given above showing that Corollary 3 holds. As such, by Corollary 3, the vector of weights $q$ derived in Eq. (2) provides a strong weighted sum representation for $\succsim$ whenever the preference order $\succsim$ satisfies Axiom 6 in addition to Axioms 1 and 2. Moreover, by Axiom 6, $e^{i} \sim e^{j}$ for all $i, j \in I$. Hence, it must be the case that $q_{i}=q_{j}$ for all $i, j \in I .^{30}$

Acknowledgements I am grateful to the editor, an associate editor, and two anonymous referees for helpful comments and suggestions, which have substantially improved the paper. I also thank Maximilian Mihm for useful comments.

Open Access This article is licensed under a Creative Commons Attribution 4.0 International License, which permits use, sharing, adaptation, distribution and reproduction in any medium or format, as long as you give appropriate credit to the original author(s) and the source, provide a link to the Creative Commons licence,

\footnotetext{
27 In general, to relate any $x$ and $y$ with $q x=q y$ and $x \neq y$, we can apply a similar argument iteratively considering at each step a distinct pair of attributes $i, j \in I$ with $x_{i}>y_{i}$ and $x_{j}<y_{j}$. Since there are finitely many attributes, the procedure would end after finitely many iterations.

28 Recall that when $q$ provides a weak representation for the DM's preference order $\succsim$, then $q$ yields a partial representation for $\succsim$ if and only if for all $x, y \in X, q x=q y$ implies $x \sim y$.

29 In an earlier work, d'Aspremont and Gevers (2002) note that adding Anonymity to Monotonicity and Translation Invariance would make all weights of the weak weighted sum representation equal, and therefore strictly positive. By contrast, Corollary 4 not only constructs the weights, but also shows that these weights would provide a strong weighted sum representation, and not just weak representation.

30 Notice that Substitutability (Axiom 3 ) is not needed for Corollary 4 since Anonymity (Axiom 6) provides a particular form of substitution across different attributes.
} 
and indicate if changes were made. The images or other third party material in this article are included in the article's Creative Commons licence, unless indicated otherwise in a credit line to the material. If material is not included in the article's Creative Commons licence and your intended use is not permitted by statutory regulation or exceeds the permitted use, you will need to obtain permission directly from the copyright holder. To view a copy of this licence, visit http://creativecommons.org/licenses/by/4.0/.

\section{A Appendix}

\section{A.1 Preliminaries}

The following lemma, which we invoke in proving our main results, shows that Translation Invariance implies a weaker form of Scale Invariance, in which the common multiplicative factor $b$ is a positive rational.

Lemma 1 Let $\succsim$ be a preference order on $X$ satisfying Axiom 2. Then, for all $x, y \in X$, $x \succsim y$ implies $\lambda x \succsim \lambda y$ for all $\lambda \in \mathbb{Q}_{++}$with $\lambda x, \lambda y \in X$.

Proof By Axiom 2, we can easily extend the preference order $\succsim$ to $\mathbb{R}^{n}$. As such, assume without loss of generality that $X=\mathbb{R}^{n}$. We first show that for all $x, y \in X$,

$$
x \succsim y \text { implies } m x \succsim m y \quad \forall m \in \mathbb{N}
$$

Clearly, (4) is true for $m=1$. Assume that it is true for $m=k$. Then, we have $k x \succsim k y$. By Axiom 2, we have $k x+x \succsim k y+x$. Also, using Axiom 2 and $x \succsim y$ we have $k y+x \succsim k y+y$. Combining these, we have $(k+1) x \succsim(k+1) y$ by transitivity of $\succsim$. This proves (4) by induction.

Next, we show that for all $x, y \in X$,

$$
x \succsim y \text { implies } r x \succsim r y \forall r \in \mathbb{Q}_{++}
$$

Given $r \in \mathbb{Q}_{++}$, we can find positive integers $s$ and $t$ such that $r=(s / t)$. Define $x^{\prime}=(x / t)$ and $y^{\prime}=(y / t)$. Note that $x^{\prime}, y^{\prime} \in X$. We claim that $x^{\prime} \succsim y^{\prime}$. For if this does not hold, then by (4) we have $t>1$, and by completeness of $\succsim$, we must have $y^{\prime} \succ x^{\prime}$. Then $(t-1) \in \mathbb{N}$ and by applying (4), we have $(t-1) y^{\prime} \succsim(t-1) x^{\prime}$. Also, using $y^{\prime} \succ x^{\prime}$ we have $t y^{\prime} \equiv y^{\prime}+(t-1) y^{\prime} \succ x^{\prime}+(t-1) y^{\prime}$ and $x^{\prime}+(t-1) y^{\prime} \succsim x^{\prime}+(t-1) x^{\prime} \equiv t x^{\prime}$ by Axiom 2 . Then, by transitivity of $\succsim$, we obtain $y=t y^{\prime} \succ t x^{\prime}=x$ contradicting the fact that $x \succsim y$. Thus, $x^{\prime} \succsim y^{\prime}$ must hold. Now, applying (4), we have $r x=(s / t) x=$ $s x^{\prime} \succsim s y^{\prime}=(s / t) y=r y$ which establishes (5).

\section{A.2 Proofs of the main results in the text}

Proof of Theorem 1 Let $\succsim$ be a given preference order satisfying Axioms 1-3. We show that vector $q \in \Delta$ defined in Eq. (2) yields a weak weighted sum representation for the preference order. 
To this end, let $z \in X$, with $q z>0$, where $q$ is defined in Eq. (2). We have to show that $z \succ 0$. Define $\delta \equiv q z$ and:

$$
P=\left\{j \in J: z_{j}>0\right\} ; N=\left\{j \in J: z_{j} \leq 0\right\}
$$

Next, we define:

$$
z_{j}^{\prime}=z_{j}+\lambda_{j} \beta_{j} \text { for } j \in N ; z_{j}^{\prime}=z_{j}-\lambda_{j} \beta_{j} \text { for } j \in P
$$

where $\beta_{j}$ is defined as in Eq. (1) for all $j \in J$, and:

$$
\lambda_{j} \equiv\left(-z_{j} / \beta_{j}\right) \text { for } j \in N ; \lambda_{j} \equiv\left(z_{j} / \beta_{j}\right) \text { for } j \in P
$$

Note that $\lambda_{j} \geq 0$ for all $j \in N$, while $\lambda_{j}>0$ for all $j \in P$. Clearly, with the definitions in (8) we have:

$$
z_{j}^{\prime}=0 \text { for all } j \in J
$$

We now proceed to pick positive rationals $r_{j}$ which are sufficiently close to the corresponding $\lambda_{j}$, for each $j \in J$. To this end, define:

$$
\rho=\min \left\{\lambda_{j} \beta_{j}: j \in P\right\} ; \mu=\min \{\rho, \delta / 2\}
$$

Note that $\rho>0$ by (6) and (8), and so $\mu>0$. Let $\varepsilon \in(0, \mu)$. Then, for each $j \in N$, one can choose $r_{j} \in \mathbb{Q}_{++}$, with $r_{j} \in\left(\lambda_{j}, \lambda_{j}+\left(\varepsilon / \beta_{j}\right)\right)$, since $\lambda_{j} \geq 0$ and $\left(\varepsilon / \beta_{j}\right)>0$. For each $j \in P$, one can choose a rational $r_{j} \in\left(\lambda_{j}-\left(\varepsilon / \beta_{j}\right), \lambda_{j}\right)$. Note that for each $j \in P$, we have (by using the definitions in (10)) $\left(\varepsilon / \beta_{j}\right)<\left(\mu / \beta_{j}\right) \leq$ $\left(\rho / \beta_{j}\right) \leq\left(\lambda_{j} \beta_{j} / \beta_{j}\right)=\lambda_{j}$ so that $r_{j} \in\left(\lambda_{j}-\left(\varepsilon / \beta_{j}\right), \lambda_{j}\right)$ is necessarily positive; that is $r_{j} \in \mathbb{Q}_{++}$for each $j \in P$.

For the positive rationals $r_{j}$ for $j \in J$ defined above, we can now proceed to define $z_{j}^{\prime \prime}$ by replacing $\lambda_{j}$ by $r_{j}$ in the definitions of $z_{j}^{\prime}$ in $(7)$, and ensure that these $z_{j}^{\prime \prime} \in(0, \varepsilon)$ for each $j \in J$. To this end, define:

$$
z_{j}^{\prime \prime}=z_{j}+r_{j} \beta_{j} \text { for } j \in N ; z_{j}^{\prime \prime}=z_{j}-r_{j} \beta_{j} \text { for } j \in P
$$

Note that (by (7) and (9)), for each $j \in N$, we have $z_{j}^{\prime \prime}=\left(r_{j}-\lambda_{j}\right) \beta_{j}$ so that, since $r_{j}>\lambda_{j}$ while $\left(r_{j}-\lambda_{j}\right)<\left(\varepsilon / \beta_{j}\right)$ for each $j \in N$,

$$
0<z_{j}^{\prime \prime}<\varepsilon \text { for all } j \in N
$$

Similarly, note that (by (7) and (9)), for each $j \in P$, we have $z_{j}^{\prime \prime}=\left(\lambda_{j}-r_{j}\right) \beta_{j}$ so that, since $r_{j}<\lambda_{j}$ while $\left(\lambda_{j}-r_{j}\right)<\left(\varepsilon / \beta_{j}\right)$ for each $j \in P$,

$$
0<z_{j}^{\prime \prime}<\varepsilon \text { for all } j \in P
$$


We now complete the definition of $z^{\prime \prime} \in X$, by defining:

$$
z_{1}^{\prime \prime}=z_{1}+\sum_{j \in P} r_{j} \alpha_{j}-\sum_{j \in N} r_{j} \alpha_{j}
$$

where $\alpha_{j}$ is defined as in Eq. (1) for each $j \in J$.

We will now demonstrate that (i) $z^{\prime \prime} \sim z$, and (ii) $z^{\prime \prime} \gg 0$. The latter, by Monotonicity (Axiom 1) will imply that $z^{\prime \prime} \succ 0$, so that the former will imply $z \succ 0$, completing the proof. It is convenient for this demonstration to define for each $i \in I$,

$$
z(i)=z_{i} e^{i}, z(i)^{\prime \prime}=z_{i}^{\prime \prime} e^{i}
$$

where $e^{i}$ is the $i$-th unit vector in $\mathbb{R}^{n}$.

Step $1\left[z^{\prime \prime} \sim z\right]$ For each $j \in N$, we have (using (11) and (15)):

$$
\begin{aligned}
z(j)^{\prime \prime} & =z_{j}^{\prime \prime} e^{j}=\left(z_{j}+r_{j} \beta_{j}\right) e^{j}=z(j)+r_{j} \beta_{j} e^{j} \\
& =z(j)+r_{j}\left[\beta_{j} e^{j}-\alpha_{j} e^{1}\right]+r_{j} \alpha_{j} e^{1}
\end{aligned}
$$

We know from Eq. (1) that $\left[\alpha_{j} e^{1}-\beta_{j} e^{j}\right] \sim 0$, and so $r_{j}\left[\beta_{j} e^{j}-\alpha_{j} e^{1}\right] \sim 0$ by Axiom 2 and Lemma 1. Then by Axiom 2, and (16) we can infer that:

$$
z(j)^{\prime \prime} \sim z(j)+r_{j} \alpha_{j} e^{1} \text { for each } j \in N
$$

Similarly, using (11) and (15),

$$
z(j)^{\prime \prime} \sim z(j)-r_{j} \alpha_{j} e^{1} \text { for each } j \in P
$$

Since

$$
z^{\prime \prime}=\sum_{j \in N} z^{\prime \prime}(j)+\sum_{j \in P} z^{\prime \prime}(j)+z^{\prime \prime}(1)
$$

we can write, using (17) and (18) and Axiom 2,

$$
\begin{aligned}
z^{\prime \prime} \sim \sum_{j \in N}\left[z(j)+r_{j} \alpha_{j} e^{1}\right]+\sum_{j \in P}\left[z(j)-r_{j} \alpha_{j} e^{1}\right] \\
+\left[z_{1}+\sum_{j \in P} r_{j} \alpha_{j}-\sum_{j \in N} r_{j} \alpha_{j}\right] e^{1} \\
=\sum_{j \in N}[z(j)]+\sum_{j \in P}[z(j)]+\left[z_{1}\right] e^{1}=z
\end{aligned}
$$

This completes Step 1 of the demonstration. 
Step $2\left[z^{\prime \prime} \gg 0\right.$ ] Using the definitions of $z^{\prime \prime}$ in (11) and (14), we can write (noting the definition of $q$ in Eq. (2)):

$$
\begin{aligned}
q z^{\prime \prime}= & \sum_{j \in N} q_{j}\left[z_{j}+r_{j} \beta_{j}\right]+\sum_{j \in P} q_{j}\left[z_{j}-r_{j} \beta_{j}\right] \\
& +q_{1}\left[z_{1}+\sum_{j \in P} r_{j} \alpha_{j}-\sum_{j \in N} r_{j} \alpha_{j}\right] \\
= & q z+\sum_{j \in N} r_{j}\left(\alpha_{j} / \sigma\right)-\sum_{j \in P} r_{j}\left(\alpha_{j} / \sigma\right)+(1 / \sigma) \sum_{j \in P} r_{j} \alpha_{j}-(1 / \sigma) \sum_{j \in N} r_{j} \alpha_{j} \\
= & q z
\end{aligned}
$$

Since $q z=\delta>0$, we then have $q z^{\prime \prime}=\delta$. Thus, using (12) and (13), we can write:

$$
\begin{aligned}
\delta & =q z^{\prime \prime}=\sum_{j \in N} q_{j} z_{j}^{\prime \prime}+\sum_{j \in P} q_{j} z_{j}^{\prime \prime}+q_{1} z_{1}^{\prime \prime} \\
& <\varepsilon\left[\sum_{j \in N} q_{j}+\sum_{j \in P} q_{j}\right]+q_{1} z_{1}^{\prime \prime} \\
& <\varepsilon+q_{1} z_{1}^{\prime \prime} \leq(\delta / 2)+q_{1} z_{1}^{\prime \prime}
\end{aligned}
$$

Clearly, (20) implies that $q_{1} z_{1}^{\prime \prime} \geq(\delta / 2)>0$, and so (using Eq. (2)), we have $z_{1}^{\prime \prime}>0$. Combining this fact with (12) and (13), we have $z^{\prime \prime} \gg 0$, completing Step 2 of the demonstration. By Axiom 1 and Step 2, we infer that $z^{\prime \prime} \succ 0$, and by Step 1 we then infer that $z \succ 0$ as desired.

Proof of Theorem 2 In view of Axiom 2, it is sufficient to show that:

$$
z \in X: z \succ 0 \text { implies } q z>0
$$

Suppose, contrary to (21), that there is $z \in X$ with $z \succ 0$, and $q z \leq 0$. By Theorem 1 , we have in fact $q z=0$. Let $r$ be an arbitrary positive real number. Define $z(r)=z+r e$ where $e=(1,1, \ldots, 1) \in \mathbb{R}^{n}$. Since $z \succ 0$, by Axiom 2 we also have:

$$
z+r e \succ 0+r e \equiv r e
$$

Let $v: X \rightarrow \mathbb{R}$ be a partial representation of the preference order $\succsim$. Then, by (22), we have:

$$
v(z(r)) \equiv v(z+r e)>v(r e)
$$

We denote:

$$
I(r)=[v(r e), v(z(r))]
$$


and note that, by (23), $I(r)$ is a non-degenerate interval in $\mathbb{R}$. Now let $\rho, \rho^{\prime}$ be arbitrary positive real numbers with $\rho^{\prime}>\rho$. Then, since $q z=0$, we have:

$$
q \rho^{\prime} e=q\left(\rho^{\prime}-\rho\right) e+q \rho e+q z=q\left(\rho^{\prime}-\rho\right) e+q z(\rho)>q z(\rho)
$$

so that:

$$
q\left(\rho^{\prime} e-z(\rho)\right)>0
$$

Using (25) and Theorem 1, we can infer that $\rho^{\prime} e-z(\rho) \succ 0$, so that by Axiom 2, $\rho^{\prime} e \succ z(\rho)$ and since $v: X \rightarrow \mathbb{R}$ is a partial representation of the preference order $\succsim$,

$$
v\left(\rho^{\prime} e\right)>v(z(\rho))
$$

From (24) and (26), we see that the interval $I\left(\rho^{\prime}\right)$ is entirely on the right of the interval $I(\rho)$ on the real line. Thus, we have a one-to-one correspondence between $\mathbb{R}_{++}$ (which is uncountable) and the collection of non-overlapping intervals $\{I(r)\}_{r \in \mathbb{R}_{++}}$, which is countable. This contradiction establishes (21) and hence the Theorem.

\section{A.3 A decomposition of the set of irrational numbers}

Using the Hausdorff Maximal Principle, we show that the following result on the decomposition of the set of irrational numbers $\mathbb{I}$ holds. ${ }^{31}$

Theorem 3 There exist subsets $\mathbb{A}$ and $\mathbb{B}$ of the set of irrationals $\mathbb{I}$ in $\mathbb{R}$, satisfying the following four properties: ( $i) \mathbb{A} \cup \mathbb{B}=\mathbb{I}$, and $\mathbb{A} \cap \mathbb{B}=\emptyset$, (ii) $\mathbb{A}=-\mathbb{B}$, (iii) if a $\in \mathbb{A}$ and $a^{\prime} \in \mathbb{A}$, then $\left(a+a^{\prime}\right) \in \mathbb{A}$, and (iv) if $a \in \mathbb{A}$ and $q \in \mathbb{Q}$, then $(a+q) \in \mathbb{A}$.

We add a few remarks to clarify the nature of the decomposition. By (i) and the uncountability of irrationals, at least one of the two sets must be uncountable. By (ii), both must be uncountable. But, properties (i) and (ii) by themselves are not of particular interest. For example, the set of positive irrationals and the set of negative irrationals will also provide a decomposition of $\mathbb{I}$ satisfying properties (i) and (ii). The properties of interest arise from (iii) and (iv), when taken in conjunction with (i) and (ii). Because of (ii), these properties hold of course for the set $\mathbb{B}$ as well; that is, we also have (iii') if $b, b^{\prime} \in \mathbb{B}$, then $\left(b+b^{\prime}\right) \in \mathbb{B}$ and (iv') if $b \in \mathbb{B}$ and $q \in \mathbb{Q}$, then $(b+q) \in \mathbb{B}$.

To appreciate (iii), note that this property is clearly not satisfied by the set of positive irrationals. For instance, $\pi$ and $(4-\pi)$ are positive irrationals, but their sum is not an irrational. Further, even though $\pi$ and $e$ are positive irrationals, the present state of knowledge about the theory of numbers does not indicate whether $(\pi+e)$ is irrational or not. ${ }^{32}$ However, the above decomposition manages to avoid these problems: if $a$

\footnotetext{
31 We would like to thank Professor Shankar Sen of the Department of Mathematics at Cornell University for his input on the decomposition result.

32 See Morandi (1996, p. 174) for a discussion of this observation.
} 
and $a^{\prime}$ are in the set $\mathbb{A}$, then not only is their sum an irrational, but it is also in the set $\mathbb{A}$. To appreciate (iv), note that given any $a \in \mathbb{A}$, the set $\{a+q: q \in \mathbb{Q}\}$ is a countable dense subset of the reals, $\mathbb{R}$. Since $\mathbb{A}$ is uncountable, $\mathbb{A}$ must contain the uncountable union of all such sets. This means the elements of the (disjoint) sets $\mathbb{A}$ and $\mathbb{B}$ are very finely interlaced along the entire real line.

In proving Theorem 3 below, we will be considering semigroups $(G, *)$, where $G$ will be a non-empty subset of the reals, and $*$ will be the binary operation of addition of reals (denoted as usual by + ). ${ }^{33}$ Moreover, we shall refer to the set itself as the semigroup, it being understood that + is the associative binary operation on the set.

Proof of Theorem 3 Consider the collection of sets:

$$
F=\{M \subset \mathbb{I}: M \text { is a semigroup }\}
$$

Note that $F$ is a non-empty collection of sets. To see this, let $a$ be any irrational and define:

$$
A(a)=\left\{z \in \mathbb{R}: z=m a+q \text { for some } m \in \mathbb{Q}_{++} \text {and } q \in \mathbb{Q}\right\}
$$

It is straightforward to verify that $A(a) \subset \mathbb{I}$ and $(A(a),+)$ is a semigroup.

Set inclusion $\subset$ is a partial ordering on $F$. By the Hausdorff Maximal Principle (see Royden (1988, p. 25), there is a maximal linearly ordered sub-collection $\mathcal{F}$ of $F .{ }^{34}$ Define:

$$
\mathbb{A}=\bigcup_{M \in \mathcal{F}} M
$$

and:

$$
\mathbb{B}=-\mathbb{A}
$$

We will verify that the sets $\mathbb{A}$ and $\mathbb{B}$ defined by (28) and (29) satisfy the four properties stated in Theorem 1. (iii).

Property (ii) follows directly from (29). We proceed with the verification of Property

Let $a, a^{\prime} \in \mathbb{A}$. Then by (28), there exist $M, M^{\prime} \in \mathcal{F}$ such that $a \in M$ and $a^{\prime} \in M^{\prime}$. Since $\mathcal{F}$ is linearly ordered, we can assume without loss of generality that both $a$ and $a^{\prime}$ belong to $M$. But then, since $M$ is a semigroup, $\left(a+a^{\prime}\right) \in M$ and thus by (28) $\left(a+a^{\prime}\right) \in \mathbb{A}$ which establishes Property (iii).

Property (iii) shows that $\mathbb{A}$ is a groupoid and therefore a semigroup. Since $\mathbb{A}$ is the union of sets which are subsets of $\mathbb{I}$, it is a subset of $\mathbb{I}$ and thus by (27), $\mathbb{A}$ belongs to

\footnotetext{
33 A semigroup is an ordered pair $(G, *)$ where $G$ is a non-empty set and $*$ is an associative binary operation on $G$; that is, a semigroup is a ordered pair $(G, *)$ such that (i) given any two elements $a, b \in G$, there is a unique element $a * b \in G$ and (ii) given any three elements $a, b, c \in G$, then $(a * b) * c=a *(b * c)$. For basic concepts of semigroup theory, see Howie (1995).

34 The Hausdorff Maximal Principle is equivalent to Zorn's Lemma [see, e.g., Kaplansky (1972, p. 60)].
} 
$F$. But then, since $M \subset \mathbb{A}$ for all $M \in \mathcal{F}$ by (28) and $\mathcal{F}$ is a maximal linearly ordered sub-collection of $F, \mathbb{A}$ is in $\mathcal{F}$. Further, $\mathbb{A}$ is a maximal element of $F$. That is:

$$
S \in F \text { with } \mathbb{A} \subset S \text { implies that } S=\mathbb{A}
$$

We now verify Property (iv). Consider the set $\mathbb{A}+\mathbb{Q}=\{z \in \mathbb{R}: z=a+q$ for some $a \in \mathbb{A}$ and $q \in \mathbb{Q}\}$. Since addition of an irrational with a rational gives an irrational, the set $\mathbb{A}+\mathbb{Q}$ is a subset of $\mathbb{I}$. Furthermore, since sets $\mathbb{A}$ and $\mathbb{Q}$ are semigroups, the set $\mathbb{A}+\mathbb{Q}$ is a semigroup. Thus $\mathbb{A}+\mathbb{Q}$ belongs to $F$. Note that since $0 \in \mathbb{Q}$ (by convention), $\mathbb{A} \subset \mathbb{A}+\mathbb{Q}$ and therefore by (30), $\mathbb{A}+\mathbb{Q}=\mathbb{A}$, establishing property (iv).

Finally, we show that Property (i) holds. The sets $\mathbb{A}$ and $\mathbb{B}$ are disjoint. Otherwise, there would exist some $z \in \mathbb{A} \cap \mathbb{B}$. So, $z \in \mathbb{A}$ and by property (ii), $(-z) \in \mathbb{A}$. But then, since $\mathbb{A}$ is a semigroup, $z+(-z)=0 \in \mathbb{A}$, a contradiction.

It remains to show that $\mathbb{A} \cup \mathbb{B}=\mathbb{I}$. Since $\mathbb{A} \subset \mathbb{I}$ (by (28)), we have $\mathbb{B}=-\mathbb{A} \subset \mathbb{I}$, and so $\mathbb{A} \cup \mathbb{B} \subset \mathbb{I}$. Suppose that there exists $x \in \mathbb{I} \backslash(\mathbb{A} \cup \mathbb{B})$. We claim that (a) there is some $\bar{m} \in \mathbb{N}$ such that $\bar{m} x \in \mathbb{B}$, and (b) there is some $\bar{n} \in \mathbb{N}$ such that $\bar{n} x \in \mathbb{A}$.

To establish (a), define the set $\mathbb{H}=\{m x: m \in \mathbb{N}\}$. Clearly, $\mathbb{H} \subset \mathbb{I}$ and $\mathbb{H}$ is a semigroup; thus, $\mathbb{H}$ is in $F$. Since $\mathbb{A}$ and $\mathbb{H}$ are semigroups, so is $(\mathbb{A}+\mathbb{H})$. Next, define the set $\mathbb{A}^{\prime}=\mathbb{A} \cup(\mathbb{A}+\mathbb{H})$. By property (iii), $\mathbb{A}+(\mathbb{A}+\mathbb{H})=(\mathbb{A}+\mathbb{A})+\mathbb{H} \subset(\mathbb{A}+\mathbb{H}) \subset \mathbb{A}^{\prime}$, and since $\mathbb{A}$ and $(\mathbb{A}+\mathbb{H})$ are semigroups, $\mathbb{A}^{\prime}$ is also a semigroup.

If $\mathbb{A}^{\prime} \subset \mathbb{I}$ then $\mathbb{A}^{\prime} \in F$. And, since $\mathbb{A} \subset \mathbb{A}^{\prime}$, we must have:

$$
\mathbb{A}^{\prime}=\mathbb{A}
$$

by (30). Define $\mathbb{G}=\mathbb{A} \cup \mathbb{H}$. Since $\mathbb{A}$ and $\mathbb{H}$ belong to $\mathbb{I}, \mathbb{G}$ also belongs to $\mathbb{I}$. Since $\mathbb{A}$ and $\mathbb{H}$ are semigroups and $(\mathbb{A}+\mathbb{H}) \subset \mathbb{A}^{\prime}=\mathbb{A} \subset \mathbb{G}$, the equality following from (31), $\mathbb{G}$ is a semigroup. Therefore $\mathbb{G}$ is in $F$. Since $\mathbb{A} \subset \mathbb{G},(30)$ implies that:

$$
\mathbb{G}=\mathbb{A}
$$

Since $x \in \mathbb{H} \subset \mathbb{G}$, we must therefore have $x \in \mathbb{A}$, a contradiction. Thus, $\mathbb{A}^{\prime}$ cannot be a subset of $\mathbb{I}$. This means there is some $q \in \mathbb{Q}$ which belongs to $\mathbb{A}^{\prime}$. Thus, there is some $a \in \mathbb{A}$ and $\bar{m} \in \mathbb{N}$, such that $(a+\bar{m} x)=q$. By property (iv), $(-\bar{m} x)=a-q \in \mathbb{A}$, and so $\bar{m} x \in \mathbb{B}$ by property (ii). This establishes claim (a).

We can establish claim (b) by applying a similar argument on $\mathbb{B}$ once we show that $\mathbb{B}$ is also a maximal element of $F$. It is straightforward to verify that for any $S \in F$, we have $(-S) \in F$. Since $\mathbb{B}=-\mathbb{A}$ by property (ii) and $\mathbb{A} \in F$, we have $\mathbb{B} \in F$. Now consider any $S \in F$ satisfying $\mathbb{B} \subset S$. Then we have, $\mathbb{A} \subset(-S)$ by property (ii) and thus $(-S)=\mathbb{A}$ by $(30)$. That is, $S=-\mathbb{A}=\mathbb{B}$ by property (ii). This shows that $\mathbb{B}$ is a maximal element of $F$. Now, applying the argument leading to claim (a), there exist $q \in \mathbb{Q}, b \in \mathbb{B}$ and $\bar{n} \in \mathbb{N}$ such that $b+\bar{n} x=q$. By property (iv'), $\bar{n} x=-b+q \in \mathbb{A}$, by properties (ii) and (iv). This establishes claim (b).

To complete the proof of property (i), note that since $\mathbb{A}$ and $\mathbb{B}$ are semigroups, we must have $(\bar{m} \bar{n}) x \in \mathbb{A} \cap \mathbb{B}$. This, however, contradicts the fact that $\mathbb{A}$ and $\mathbb{B}$ are disjoint sets. Thus, we must have $\mathbb{A} \cup \mathbb{B}=\mathbb{I}$. 


\section{References}

Banerjee, K., Mitra, T.: On Wold's approach to representation of preferences. J. Math. Econ. 79, 65-74 (2018)

Birkoff, G.: Lattice Theory. American Mathematical Society Publications, New York (1948)

Blackwell, D., Girshick, M.A.: Theory of Games and Statistical Decisions. Wiley, New York (1954)

Bridges, D.S., Mehta, G.B.: Representation of Preference Orderings. Springer, Berlin (1995)

Candeal, J.C.: Invariance axioms for preferences: applications to social choice theory. Soc. Choi. Welf. 41, 453-471 (2013)

Candeal, J., Indurain, E.: A note on linear utility. Econ. Theory 6, 519-522 (1995)

Chipman, J.S.: The foundations of utility. Econometrica 28, 193-224 (1960)

d'Aspremont, C.: Axioms for social welfare orderings. In: Hurwicz, L., Schmeidler, D., Sonnenschein, H. (eds.) Social Goals and Social Organization. Essays in Memory of Elisha Pazner, pp. 19-75. Cambridge University Press, Cambridge (1985)

d'Aspremont, C., Gevers, L.: Equity and the informational basis of collective choice. Rev. Econ. Stud. 44, 199-209 (1977)

d'Aspremont, C., Gevers, L.: Social welfare functionals and interpersonal comparability. In: Arrow, K., Sen, A., Suzumura, K. (eds.) Handbook of Social Choice and Welfare, pp. 459-541. Elsevier, Amsterdam (2002)

Debreu, G.: Representation of a preference ordering by a numerical function. In: Thrall, R.M., Coombs, C.H., Davis, R.L. (eds.) Decision Processes, pp. 159-165. Wiley, New York (1954)

Fleurbaey, M., Maniquet, F.: A Theory of Fairness and Social Welfare. Cambridge University Press, Cambridge (2011)

Gevers, L.: On interpersonal comparability and social welfare orderings. Econometrica 47, 75-89 (1979)

Hara, K., Ok, E.A., Riella, G.: Coalitional expected multi-utility theory. Econometrica 87, 933-980 (2019)

Hausner, M., Wendel, J.: Ordered vector spaces. Proc. Am. Math. Soc. 3, 977-982 (1952)

Howie, J.M.: Fundamentals of Semigroup Theory. Clarendon Press, Oxford (1995)

Kaplansky, I.: Set Theory and Metric Spaces. Chelsea, New York (1972)

Krause, U.: Essentially lexicographic aggregation. Soc. Choice Welf. 12, 233-244 (1995)

Mehta, G.B.: Preference and utility. In: Barbera, S., Hammond, P., Seidl, C. (eds.) Handbook of Utility Theory, vol. 1. Kluwer, Dordrecht (1998)

Mitra, T., Ozbek, M.K.: On Representation of monotone preference orders in a sequence space. Soc. Choice Welf. 41, 473-487 (2013)

Mongin, P., d'Aspremont, C.: Utility theory and ethics. In: Barberà, S., Hammond, P., Seidl, C. (eds.) Handbook of Utility Theory, pp. 371-481. Kluwer, Boston (1998)

Morandi, P.: Field and Galois Theory. Springer, Berlin (1996)

Moulin, H.: Axioms of Cooperative Decision Making. Cambridge University Press, Cambridge (1988)

Neuefeind, W., Trockel, W.: Continuous linear representability of binary relations. Econ. Theory 6, 351-356 (1995)

Roberts, K.W.S.: Interpersonal comparability and social choice theory. Rev. Econ. Stud. 47, 421-439 (1980)

Royden, H.L.: Real Analysis. Macmillan, New York (1988)

Segal, U., Sobel, J.: Min, max, and sum. J. Econ. Theory 106, 126-150 (2002)

Trockel, W.: An alternative proof for the linear utility representation theorem. Econ. Theory 2, 298-302 (1992)

Weibull, J.W.: Discounted value representations of temporal preferences. Math. Oper. Res. 10, 244-250 (1985)

Yoshihara, N., Veneziani, R.: The Measurement of Labour Content: A General Approach. Mimeo, New York (2018)

Publisher's Note Springer Nature remains neutral with regard to jurisdictional claims in published maps and institutional affiliations. 